Relations industrielles

Industrial Relations

\title{
Relations professionnelles, emploi et formation au Québec
}

\section{Christian Maroy}

Volume 50, numéro 4, 1995

L'ergonomie et les relations industrielles

Ergonomics and Industrial Relations

URI : https://id.erudit.org/iderudit/051063ar

DOI : https://doi.org/10.7202/051063ar

Aller au sommaire du numéro

Éditeur(s)

Département des relations industrielles de l'Université Laval

ISSN

0034-379X (imprimé)

1703-8138 (numérique)

Découvrir la revue

Citer ce compte rendu

Maroy, C. (1995). Compte rendu de [Relations professionnelles, emploi et formation au Québec]. Relations industrielles / Industrial Relations, 50(4), 895-898. https://doi.org/10.7202/051063ar

Tous droits réservés @ Département des relations industrielles de l'Université Laval, 1995
Ce document est protégé par la loi sur le droit d'auteur. L'utilisation des services d'Érudit (y compris la reproduction) est assujettie à sa politique d'utilisation que vous pouvez consulter en ligne.

https://apropos.erudit.org/fr/usagers/politique-dutilisation/ 
case against North American adversarialism, Adams makes much of the superior rates of productivity growth in Europe and Japan from 1960 to 1990. Yet there is no consistent pattern if one looks at his data for the 1980s (p. 136), even though, according to Adams, nonadversarial systems had major restructuring and hence performance advantages during this period. Moreover, based upon purchasing power comparisons, the U.S. still had the highest level of productivity per person employed of any liberal democracy as of 1992 , while Canada ranked anywhere from second to fourth in the world, depending upon the method of calculation employed. Thus, whether "nonadversarial" systems actually do give rise to superior economic performance is unclear.

These are, however, largely paradigmatic issues, and they are not in any way devastating to the book. Instead, they suggest only that this book should be read critically and supplemented with other sources if adopted for use in the class room. As an introduction to comparative IR from a North American perspective, this book is highly worthwhile, serving to provoke as well as inform. In particular, it cuts through the maze most North Americans typically face when attempting to study alternative IR systems. This is not an easy task, and the author as a result focuses primarily upon the differences between the noncorporatist systems of the U.S. and Canada, and the neocorporatist systems of Sweden and Germany. But, at minimum, this book provides an excellent foundation for subsequent, more in-depth study of comparative IR systems.

To conclude, Adams does a great job of analyzing the essential differences between North American and other liberal democratic IR systems, and, more important, of illustrating why these differences are of relevance to North Americans. As a result, this book should not only be of value in the class room, it should also help to stimulate greater interest in comparative IR in both academic and policy circles. The author has made an important contribution, and is to be congratulated.

JOHN GODARD

University of Manitoba

Relations professionnelles, emploi et formation au Québec

Critique Régionale, $\mathrm{n}^{\text {os }} 23-24,1994$, Université Libre de Bruxelles, 161 p., ISSN 0770-0075.

C'est comme lecteur européen, familier sans être expert de la réalité québécoise, que j'ai abordé cette publication. Dans son introduction en effet, les responsables - québécois - de cette livraison de Critique Régionale précisent que son but est "de fournir aux lecteurs européens une vision et de la réalité du monde du travail au Québec et des recherches qui s'y font ". On peut d'emblée souligner que le but est pour l'essentiel rempli. Celui qui ne connaît en rien le système de relations industrielles québécois, celui qui s'interroge sur les évolutions des grands paramètres de l'emploi ou souhaite saisir les grandes lignes des politiques menées à l'endroit de l'enseignement ou de la formation professionnelle y trouvera un panorama large et documenté. Si certains volets paraissent moins développés - on aurait souhaité une synthèse des travaux quantitatifs menés dans le domaine de l'insertion professionnelle des jeunes ou des sans-emploi -, si d'autres sont interrogés moins sur le plan factuel que celui des problèmes d'analyse qu'ils génèrent (l'évolution de l'organisation du travail), il n'en demeure pas moins que, globalement, le numéro alimente une réflexion intéressante et présente une vue d'ensemble sur le Québec en ces matières. 
Lise Poulin Simon et Diane-Gabrielle Tremblay nous présentent d'abord un tableau quantitatif des évolutions du marché du travail, caractérisé au Québec par un chômage élevé et le développement des formes d'emplois précaires. Par la suite, elles tracent avec clarté les grandes étapes et les inflexions des politiques de l'emploi et d'aide sociale. Leur propos est surtout de montrer combien les politiques "généreuses " des années 70 sont remises en cause. On passe ainsi des politiques "passives" aux politiques dites "actives". Les auteures concluent notamment sur la nécessité d'une politique "active" plus cohérente, en souhaitant voir l'ensemble des compétences en matière de formation revenir à la province de Québec. Par contre, elles n'interrogent guère la notion et la pertinence même des politiques actives, qui ont des limites si elles ne sont pas envisagées avec d'autres mesures favorisant la création et le partage des emplois. On aurait aimé voir l'argumentation des auteures à ce sujet.

L'article de Gregor Murray et Pierre Verge constitue un état des lieux synthétique et argumenté de la situation du mouvement syndical au Québec et au Canada. Après avoir rappelé les particularités nord-américaines des relations industrielles, ils présentent l'évolution quantitative des taux de syndicalisation et des taux de présence syndicale, soulignant l'évolution divergente à cet égard entre les États-Unis (forte décroissance) et le Canada ou le Québec (légère croissance sauf dans les services privés). Ils soulignent ensuite l'orientation, les forces et les faiblesses de l'action professionnelle et de l'action politique des syndicats au Québec et les mettent en contexte. On ne peut que souligner la qualité et la pertinence des informations présentées, la qualité de la discussion engagée à leur propos. J'épinglerai seulement deux éléments particulièrement stimulants de celle-ci: d'une part, les auteurs soulignent que la prédominance de négociations locales au niveau de l'entreprise et la forte présence syndicale au Québec pourraient constituer des conditions favorisant leur capacité à négocier, en faveur des travailleurs, la modernisation des modèles de gestion de l'entreprise, comme cela s'est passé dans quelques cas encore très minoritaires. Ainsi, des traits qui, par ailleurs, peuvent être des faiblesses (défavorisant la cohésion d'ensemble du mouvement) peuvent aussi représenter une force. D'autre part, ils discutent l'institutionnalisation relative du mouvement syndical et sa présence au niveau politique; ils en montrent bien le caractère "hybride " et "incertain ". Cependant, on aurait aimé qu'ils en discutent davantage les conséquences sur la capacité du mouvement syndical à influencer les politiques macro-économiques, au moment où le Canada et le Québec accentuent les programmes de résorption des déficits de l'État et de la sécurité sociale.

Mona-Josée Gagnon présente les recherches sur l'évolution de l'organisation du travail dans les entreprises québécoises. Elle insiste surtout sur les limites de ces recherches. Limites des diagnostics globalisants alors que les résultats empiriques s'appuient sur des monographies éparses et souvent " exceptionnelles". Limites des approches engagées (école de la régulation "sociologisée ", école des choix stratégiques...) dont il lui paraît douteux de "voir émerger un nouveau paradigme explicatif". Elle insiste quant à elle sur la variété des situations concrètes même si des constantes (l'implication, la flexibilité) apparaissent au niveau des projets managériaux. Le diagnostic de Gagnon est fort sévère à l'égard de l'apport théorique et empirique des recherches québécoises. Certes, sa mise en garde contre les généralisations abusives à partir de cas médiatisés est salutaire. Néanmoins, on ne la voit guère esquisser de pistes théoriques alternatives, ni comment on pourrait faire l'économie de la multiplication et comparaison critique des études de cas. 
Colette Bernier fournit une contribution stimulante mettant en relation les caractéristiques du régime de relations industrielles (accréditation de syndicats d'établissements, importance de l'ancienneté) et la prégnance d'un modèle traditionnel de formation de la main-d'œuvre au Québec. Ce modèle privilégie la formation en entreprise, sur le tas, courte et peu qualifiante. Elle montre ensuite comment la formation professionnelle a eu tendance à s'institutionnaliser à la fin des années 80 via une politique visant la promotion de la concertation (commissions paritaires de formation professionnelle: CFP) et le recours accru au système d'enseignement dans l'utilisation des fonds accordés par le gouvernement fédéral aux entreprises. Or cette politique incitative (illustrée avec le cas du secteur de l'aérospatiale) n'a pas suffi à développer l'effort de formation de la main-d'œuvre : la formation de la relève a été privilégiée et la concertation n'a pas gagné l'intérieur de l'entreprise. D'où la nécessité d'une loi plus contraignante pour développer la formation de la maind'œuvre. Cette analyse est assurément stimulante. Elle inviterait cependant à une analyse comparative plus approfondie avec d'autres situations nationales. Le modèle "traditionnel " de formation de la main-d'œuvre occupée est partagé par d'autres pays où des régimes différents de relations industrielles sont en place. Par ailleurs, la France, dotée d'une loi contraignante en matière de formation, a assurément augmenté l'effort de formation en direction des salariés, sans pour autant que les formations "innovantes" ne soient devenues la règle.

Christian Payeur met bien en évidence les grandes étapes et les traits du développement du système d'enseignement au Québec ; il souligne la place de la formation professionnelle en son sein. Le Québec, comme d'autres pays industrialisés, après avoir promu l'égalisation des chances et le développement d'un enseignement élevant le niveau de for- mation générale de tous, développe depuis la fin des années 80 ce qu'on pourrait appeler, comme au RoyaumeUni, une "nouvelle professionnalisation de l'enseignement "(new vocationalism). Son propos est alors de souligner avec clarté les problèmes et les enjeux posés par les réformes successives depuis 1985 : faiblesse persistante des effectifs jeunes en formation professionnelle, décrochage scolaire et risques de nouvelles exclusions, incohérences des politiques pour les 16-19 ans, contradictions de nouvelles mesures d'apprentissage notamment. Les jugements qu'il porte impliquent d'évidence des choix politiques et normatifs, notamment quant à la nécessaire place centrale des institutions scolaires dans les dispositifs ; ils sont néanmoins largement étayés par les données empiriques qu'il rapporte.

Les textes de Claude Laflamme et Pierre Paillé d'une part, celui de Nicole Lemieux d'autre part, rendent compte de recherches qualitatives dans le domaine de l'insertion professionnelle. Les premiers s'intéressent au processus de socialisation organisationnelle, dans des PME, de jeunes pour l'essentiel sans diplôme. Bien qu'ils ne définissent pas explicitement le concept de socialisation, leur article montre à quel point les typologies anglo-saxonnes s'avèrent caduques pour rendre compte de ce qui se passe dans ces PME. L'intégration au travail (terme équivalent apparemment à celui de socialisation) serait plutôt compréhensible "comme une logique de classe ". Le jeune doit se conformer à diverses attentes qui prennent sens par rapport à des exigences de production. Il doit aussi se faire accepter par le groupe de pairs. De tels résultats me semblent rester à la surface des processus mis en jeu; les auteurs insistent sur l'importance de "l'informel " mais on aurait aimé savoir davantage comment cet "informel " se structure et se joue. Le texte de Nicole Lemieux va nettement plus loin à cet égard. Il analyse de façon très fine les processus d'insertion 
professionnelle de jeunes filles impliquées dans divers modes de formation au secrétariat. Mettant en œuvre une démarche de terrain inspirée de la nouvelle école de Chicago, elle aboutit à une " théorie émergente " selon laquelle l'insertion professionnelle est un processus de construction de l'identité professionnelle. Son issue ne dépend pas que de la personne et de son passé mais aussi des incidents de parcours, des opportunités réelles d'emploi auxquelles les stages conduisent. Nicole Lemieux construit une typologie des modes d'insertion observés qu'elle associe à une typologie des identités: identité de métier ou d'entreprise versus identité de substitution. Le plus frappant dans ce travail intéressant est que le travail est et reste une référence essentielle dans la construction de l'identité sociale.

Enfin, Louise Boivin évalue l'évolution de la situation des femmes sur le marché du travail. Son propos souligne que les mesures en leur faveur ne portent pas tous les fruits attendus. En effet, les travailleuses restent cantonnées dans des secteurs traditionnellement " féminins " et dans des postes peu valorisés. De plus, au moment de l'introduction des nouvelles technologies, elles se font parfois exclure par suppression des emplois les moins qualifiés. Cet article est autant un constat de la situation qu'une dénonciation et un appel à y remédier.

En définitive, le lecteur européen, mais aussi québécois ou canadien, trouvera dans ce numéro une excellente introduction à la situation - et dans une moindre mesure à la recherche - québécoise concernant l'emploi, le travail et la formation professionnelle.

\section{CHRISTIAN MAROY Université Catholique de Louvain}

\section{The Humanized Workplace}

by Jerome BRAUN, Westport, CT, Praeger, 1995, 175 p., ISBN 0-275-94915-X.

The words that come most readily to mind upon reading this book are "hodgepodge," and "interesting." This book is comprised largely of what appears to be a series of essays written at different times for different purposes. The connections among them are not always apparent, and there is a good deal of repetition. Yet, sprinkled into this hodgepodge are some intriguing, and perhaps even brilliant, observations. Whether the value of these gem-like parts outweighs the pain of working through the more ordinary whole, is a matter to be judged by each reader.

As the theme of the volume is the humanized workplace in which industrial justice prevails, I will attempt in this review to concentrate on outlining and commenting upon the author's arguments on this subject. A good deal of his analysis, in a variety of ways and places. has to do with stating how to achieve justice in the workplace.

In the Preface, Braun states that competition, so important in American society, is unjust "when one side, for small benefits, expects the other side to suffer great costs." In the first chapter, he draws from a lengthy summary of a book by another author the interesting observation that American unions and managements should concern themselves not so much with the details of means, such as grievance procedures, but instead focus on ends, "eliminating false values and shortsightedness" that prevent attaining industrial justice. Early in the volume, and many times thereafter, Braun argues that fairness is an important, but lost, value in the employment relationship.

Also early in the volume, in Chapter 1 , the author launches into a vigorous 